\title{
A BANDWidth Allocation Model Provisioning FrameWork WiTH AUTONOMIC CHARACTERISTICS
}

\author{
Rafael F. Reale ${ }^{1}$, Romildo M. da S. Bezerra ${ }^{2}$ and Joberto S. B. Martins ${ }^{3}$ \\ ${ }^{1}$ Federal Institute of Bahia (IFBA) - Brazil \\ ${ }^{2}$ Federal Institute of Bahia (IFBA) - Brazil \\ ${ }^{3}$ Computer Networks Research Group (NUPERC) - Salvador University (UNIFACS) - Brazil
}

\begin{abstract}
The Bandwidth Allocation Models (MAM, RDM, G-RDM and AllocTC-Sharing) are management alternatives currently available which propose different resource (bandwidth) allocation strategies in multiservice networks. The BAM adoption by a network is typically a management choice and configuration task executed by the network operations and management system setup in a static or nearly static way. This paper proposes and explores the alternative of allowing BAM definition and configuration on a more dynamic way. In effect, one of the basic motivations towards BAM dynamic allocation is the fact that multiservice networks characteristics (traffic load) may change considerably in daily network operation and, as such, some dynamics in BAM allocation should be introduced in order to improve performance. A framework is presented supporting BAM dynamic allocation. The framework adopts an OpenFlow-based software-defined networking (SDN) implementation approach in order to support scalability issues with a centralized controller and management network view. The framework architecture also supports the implementation of some autonomic characteristics which, in brief, look for improving and facilitating the decision-making process involved with BAM provisioning in a multiservice network. A proof of concept is presented evaluating different BAM performance under different traffic loads in order to demonstrate the framework strategy adopted.
\end{abstract}

\section{KEYWORDS}

Bandwidth Allocation Models (BAM), Autonomic Management, Software Defined Networking (SDN), OpenFlow, Resource Provisioning, MAM, RDM, G-RDM, AllocTC-Sharing.

\section{INTRODUCTION}

The multiservice networks are a class of computer network infrastructure in which several services and applications are integrated. Multiservice networks use a common network infrastructure and enable a more efficient use of network resources. In recent years there has been a rapid increase in the number of multimedia applications running over IP creating a significant variety of new services and applications, typical of a multiservice network. These new applications and services require some guarantee of quality of service (QoS) which are usually defined in Service Level Agreements (SLAs)[1]. The scarcity of resources and/or dynamics of the traffic generate conflicts when provisioning resources, e.g. bandwidth, in the operation of a multiservice network. As such one important and challenging aspect of the multiservice networks consists in modeling and provisioning the available resources such as bandwidth and routes among others.

This paper presents a framework which supports the provisioning of BAMs in a network, typically multiservice, with autonomic characteristics. The main motivation behind the framework and architectural decisions adopted is the need for a more dynamic management

DOI : $10.5121 /$ ijcnc.2013.5606 
solution for this area, considering that networks will continue to evolve with hundreds of possible multimedia applications with distinct SLAs and thousands of users which, in principle, want the best possible support from the network in all scenarios. In brief, an ongoing effective and complex management challenge. The framework, in terms of its implementation, also explores the new paradigm provided by OpenFlow and SDN, by adopting this protocol as the basis for communication between the framework and equipment on the network.

The paper is organized as follows. In section 2 are summarized the basic Bandwidth Allocation Models currently available. Section 3 and 4 present the motivation and problem discussion in relation to the management of BAMs in a multiservice network. Section 5 details the architecture and functionalities of a framework under development supporting the BAM provisioning. Section 6 discuss the OpenFlow-based framework implementation and section 7 presents a comparative evaluation of BAM models in order to demonstrate the need of the framework for BAM provisioning. Concluding remarks are presented in section 6.

\section{BANDWIDTH Allocation Models (BAM)}

One of the techniques that may be used to define rules and limits for link utilization for flow aggregates (traffic classes - TCs) is the Bandwidth Allocation Model (BAM).

The bandwidth allocation model (BAM) defines the rules that result in granting, blocking or preemption of a flow on a particular link. These models are associated and depend on the path selection algorithm (OSPF, CSPF, other) which defines the links in a path used by all flows. An adequate choice of the bandwidth allocation model can directly lead to an improved performance of the network as a whole as well as in meeting QoS requirements defined the SLAs.

There are alternative bandwidth allocation models such as: the Maximum Allocation Model (MAM), Russian Doll Model (RDM), Generalized-RDM (RDM-G) and AllocTC-sharing that will be shortly described next.

\subsection{Maximum Allocation Model (MAM)}

The Maximum Allocation Model (MAM) is the basic BAM model. Its main objective is to reserve a maximum bandwidth for each traffic class (TC) by mapping a bandwidth constraint (BC) to the traffic classes (TCs)[5].

MAM model can be described as follows:

1) For each Traffic Class "TCi" where "M" is the maximum reservable bandwidth on link and "Ni" is the bandwidth allocated for TC have:

$$
\mathrm{Ni} \leq \mathrm{BCi} \leq \mathrm{M}
$$

2) With the restrictions, the total bandwidth allocated by the TCs may not exceed the link capacity:

$$
\left(\sum_{i=0}^{C-1} N i \leq M\right)
$$

In this way, the sum of the total allocated bandwidth occupied by flow (Ni) of a particular TC should always be less than or equal to the maximum bandwidth constraint (BC) associated with this TC for a particular link. Moreover, the sum of bandwidth allocations of TCs always corresponds to the bandwidth available for allocation on link considered (Figure 1). 
International Journal of Computer Networks \& Communications (IJCNC) Vol.5, No.6, November 2013

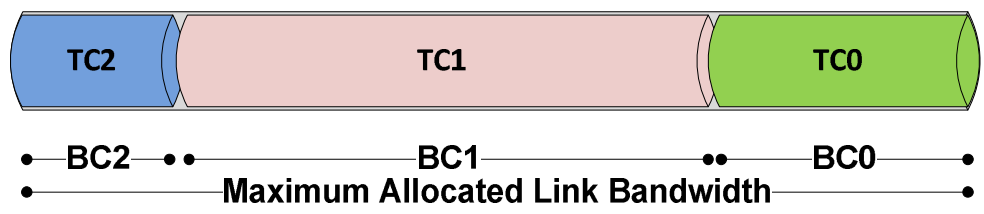

Figure 1. Maximum Allocation Model (MAM)

The inherent advantage of MAM Model is the total isolation between traffic of different TCs.

\subsection{Russian Doll Model (RDM)}

The RDM model upgrade in relation to MAM model corresponds briefly in allowing the sharing of non-used bandwidth allocated for high-priority classes by low-priority classes (Figure 2)[4]. As such, free bandwidth allocated by the manager to higher priority classes (applications) can be temporarily used by lower priority classes (applications).

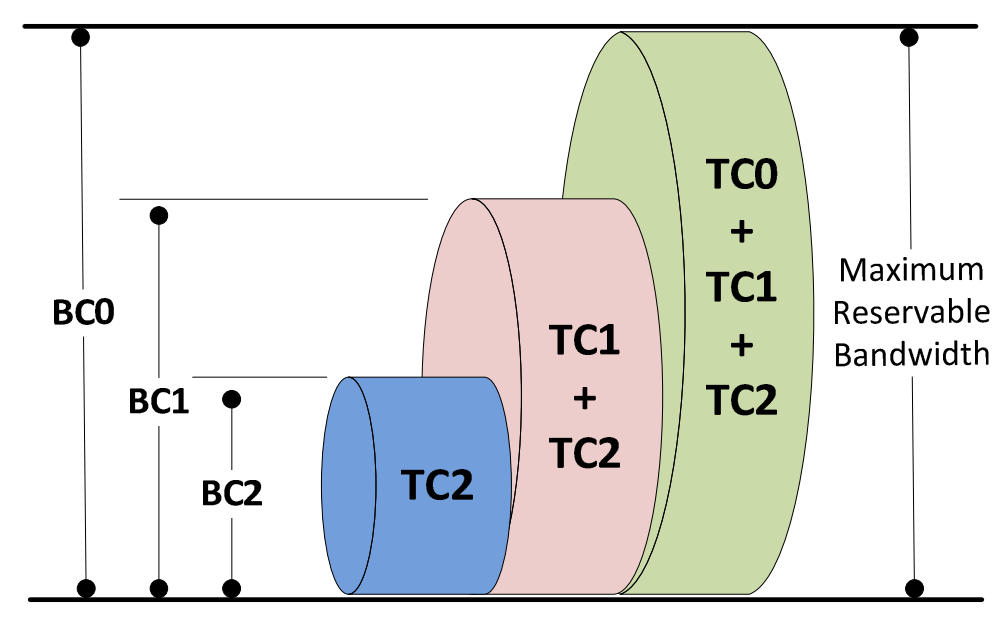

Figure 2. Russian Dolls Model (RDM)

In general RDM leads to an improved link utilization and optimization when compared with MAM model [12].

\subsection{G-RDM}

The Generalized-RDM (G-RDM) model is in effect an MAM and RDM hybrid by proposing to join the best of both models [2]. G-RDM creates the concept of shared bandwidth and private bandwidth for TCs. The private bandwidth (pools) form the reserved bandwidth class that uses MAM model and, as such, cannot be shared by other TCs. Common bandwidth pools are shared by TCs accordingly with the RDM model (Figure 3). 
International Journal of Computer Networks \& Communications (IJCNC) Vol.5, No.6, November 2013

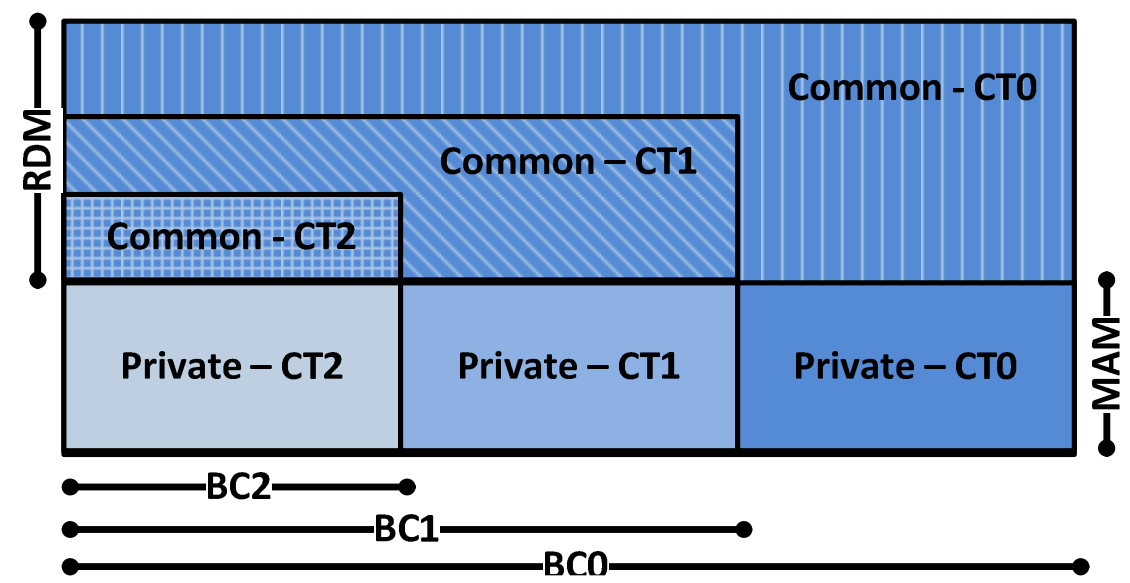

Figure 3. G-RDM (Generalized-RDM)

\subsection{AllocTC-Sharing}

The AllocTC-Sharing is another bandwidth allocation model in which all the available bandwidth is shared opportunistically among traffic classes (TCs)[9].

The AllocTC-Sharing model implements two integrated styles of sharing bandwidth (Figure 4 and Figure 5):

- Sharing "high-to-low" (high-to-low - HTL) and

- Sharing "low-to-high" (low-to-high - LTH).

Sharing "high-to-low" is the classic style of sharing used in networking applications and follows RDM model As such, unused bandwidth reserved for flows in high-priority classes are temporarily allocated to applications in lower priority classes.

Sharing "low-to-high" allows flows of high-priority class to allocate unused bandwidth reserved for flows of low-priority classes. This particular strategy is called "bandwidth loan".

In both styles AllocTC-Sharing uses a control mechanism to preserve the bandwidth restrictions set for the links. The "high-to-low" sharing makes use of "preemption" to comply with these constraints and the "low-to-high" sharing makes use of "devolution" (loan devolution).

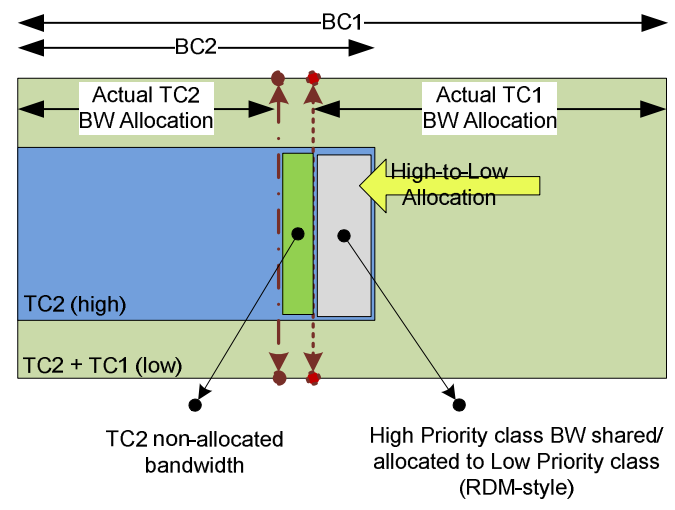

Figure 4. AllocTC-Sharing "High-to-Low" Bandwidth Allocation 


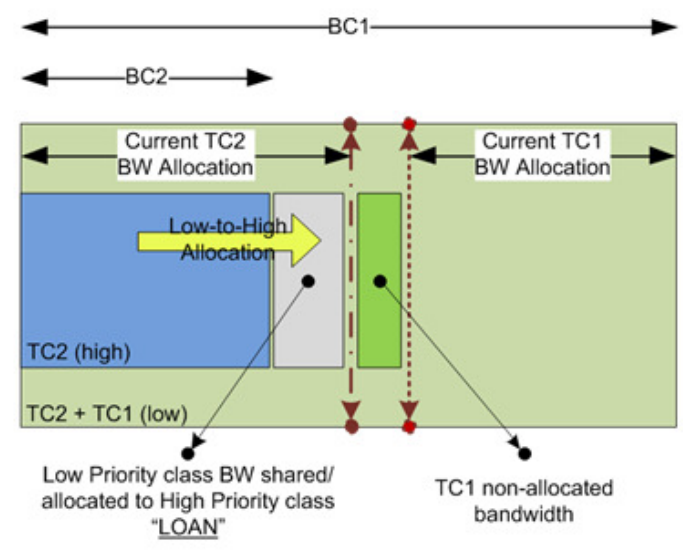

Figure 5. AllocTC-Sharing "Low-to-High" Bandwidth Allocation Figure 6.

\section{Motivation - BandwidTh Allocation Models In Networks With DYNAMIC CHARACTERISTICS}

Over the years, computer networks were designed as a set of devices centrally coordinated by the global vision of a manager or network administrator. The manager is responsible for maintaining the operation of the infrastructure, usually complex, with heterogeneous device capabilities which, in turn, support services and applications with different SLAs.

The complexity of network equipment management coupled with the growing need for service availability, directly impacts the efficiency of managers and administrators [3]. Typically, a high level of expertise and efficiency is required and this can make the human intervention a point of failure. Moreover, the operational cost is affected since, normally, a larger search time is necessary for obtaining solutions to the problems presented for the network manager and support system. Another relevant factor in this context is the need for a large amount of expertise by administrators for the effective management of complex environments.

In this same context, the bandwidth allocation models (BAMs) constitute an important and necessary functionality for network management [11]. The adoption and / or use of a specific bandwidth allocation model (MAM, RDM, G-RDM, AllocTC-Sharing, other) by the network requires constant monitoring of network parameters and administrators with high degree of expertise. In fact, the correct choice of a model and its correct configuration directly imply in compliance or not with quality of service (QoS) requirements defined by SLAs (Service Level Agreement).

The first challenge for the network administrator is to define the best bandwidth allocation model in relation to QoS requirements considering the entire network and links individually. After this step, which requires expertise in relation to the existing models and their operation, is necessary to configure the BAM operation by setting parameters and aspects such as the number of classes, flows that are associated with each class and restrictions of bandwidth for each class, among others parameters.

The decision about the bandwidth allocation model adopted is taken, usually according to the actual network baseline and, typically, decisions and resulting configurations have a static 
characteristic, as far as it is only adjusted when the administrator network decides it is necessary. We have then a scenario of complex decision in a network management structure that is nearstatic.

In general, we understand that computer network management should be more dynamic and, moreover, that the history of the network operation, your current scenario and its future projection should be taken into account in deciding about the bandwidth allocation model adopted and its parameters [3][6].

Regarding the dynamics of the network, we argue that reconfiguration should be dynamic and, to the extent possible, "on-the-fly" so that the BAM provisioning with its parameters can evolve with the operational state of the network.

\section{The Problem Of Choosing Bandwidth Allocation Models In MULTISERVICE NETWORK}

Each bandwidth allocation model has some particular characteristics, optimizing network performance metrics, such as: link utilization, number of preemptions, new flow allocation blocking probability and number of flows treated, among others.

Thus, the administrator's choice towards a specific bandwidth allocation model mechanism adoption for a multiservice network must consider, minimally, two technical issues:

- The BAM inherent technical characteristics and optimization focus which, in turn, may define its applicability or not depending of the network management requirements considered, and

- The characterization of the multiservice traffic allocated in the network.

These technical aspects are further discussed in the next section.

\subsection{Technical and Optimization Characteristics of Bandwidth Allocation Models (BAM)}

The choice of a bandwidth allocation model and its configuration parameters is directly related to the inherent technical characteristics of the BAM adopted and, in addition, strongly depends of traffic profile resulting from the applications running on the network.

The MAM model is indicated when we have a network traffic profile where "application classes" (Traffic Classes - TCs) do not affect each other significantly. As can be seen in Table I, the model does not admit any bandwidth sharing between classes ("high to low" or "low to high"). Another aspect to consider is that the adoption of the MAM model may imply in low link utilization and high number of flow blocking. That occurs since the available resources (not allocated per TC) cannot be shared by definition among classes in MAM's model (full isolation between TCs).

The RDM model has as its basic characteristics the sharing of unallocated bandwidth resources unused by high-priority applications by low-priority applications (Table I). Thus, the RDM model is mostly suitable for networks with a traffic profile in which the low-priority applications can benefit from idle bandwidth that would be normally allocated for high-priority applications. In situations where the dynamics of network leads the high-priority applications bandwidth demand to exceed its configured capacity, new flows are blocked, even when there is bandwidth for lowpriority applications. As such, link utilization is not maximized with this BAM. 
The G-RDM model basic characteristics is to preserve MAM modeling for a reserved pool of resources (private pool) and used the RDM modeling approach for another set of allocated bandwidth resources (common pool). The G-RDM model can be consequently applied in both scenarios of traffic profile suitable for MAM and RDM. The difference in relation to each individual model is that the G-RDM bandwidth sharing is partial (part of the bandwidth resources allocated per link by the manager).

The AllocTC-sharing model as its basic characteristics the simultaneous support for both highpriority and low-priority traffic classes (TCs). In effect, the model attempts to use the available link capacity in both directions ("high to low" and "low to high") in order to maximize link utilization. AllocTC-sharing model has as its main disadvantage the need to return borrowed bandwidth (in both senses). Since high-priority TCs may use bandwidth borrowed from lowpriority TCs, high-priority application may be preempted. As such, this model is most suitable for elastic and bandwidth eager high-priority applications.

Table 1. Technical Characteristics Comparison of Bandwidth Allocation Models MAM, RDM, AllocTC-Sharing and G-RDM

\begin{tabular}{|l|l|l|l|l|}
\hline BAM Characteristics & MAM & RDM & $\begin{array}{l}\text { AllocTC- } \\
\text { Sharing }\end{array}$ & GRDM \\
\hline Sharing from "high to low" & no & yes & yes & partial \\
\hline Sharing from "low to high" & no & no & yes & no \\
\hline $\begin{array}{l}\text { Efficient bandwidth utilization } \\
\text { with high traffic (low priority) }\end{array}$ & low & high & high & medium \\
\hline $\begin{array}{l}\text { Efficient bandwidth utilization } \\
\text { with high traffic (high priority) }\end{array}$ & low & low & high & low \\
\hline Isolation between TCs & high & medium & low & medium \\
\hline
\end{tabular}

\subsection{Multiservice Traffic Characteristics}

The multiservice network traffic characterization has been studied and discussed in the literature and, for the purpose of discussing BAM evaluation impacts, it has the following basic characteristics: dynamic, asymmetrical and multi-path.

The dynamic characteristic of network traffic results from multiple applications running on top of the network with different SLAs. Beyond that, each individual application's traffic profile may change from time to time: day/night, week/weekend, holidays, others. Application's traffic is, most of the time, asymmetric due to client-server characteristics of traffic and, finally, applications make use normally of multi-path links and/or trajectories (paths).

With respect to the allocation of traffic per link which is the focus of BAM models, the nonuniform traffic per link must be considered. Non-uniform traffic per link means that it is usual to have very different traffic profiles for each individual link. This characteristic implies on the need to consider the BAM mechanism which is provisioning bandwidth for applications not only at network level but also at link level.

\subsection{Why Dynamic BAM Utilization}

Networks and their traffic profile are dynamic. In turn, distinct bandwidth allocation models are suitable for different traffic profile. As such, BAMs used by the network should also be dynamically defined, provisioned and configured in order to evolve with the actual dynamics of the network. 
As such it is necessary to have a framework with the capability to dynamically decide which bandwidth allocation model is currently adequate for a given network traffic state. Furthermore, to the extent that the decision-making process involves many parameters and on-the-fly computing, to incorporate some autonomic characteristics in the framework is also desirable. In doing this we get a more robust and less susceptible to errors solution even in the scenario of dynamic and complex decision making.

Another aspect inherent in the realization of a framework with autonomic features is the choice of the architecture adopted for the decision making process. In the proposed framework, we will focus on the choice of a bandwidth allocation model that considers the entire network as its traffic scenario. As such, we choose an architecture in which the network intelligence is centralized in terms of the framework. In effect, the framework maintains a global view of the network and management control is maintained in a single logical element (centralized management). This allows, rather than configuring each device separately, abstracting the network as a whole through the framework.

\section{A Framework for Selection and Provisioning of Bandwidth Allocation Models (BAM)}

The framework for selection of Bandwidth Allocation Models (BAM) is composed by four basic functional modules: Monitoring Module, Knowledge Module, Execution Module and a database as presented in Figure 6.

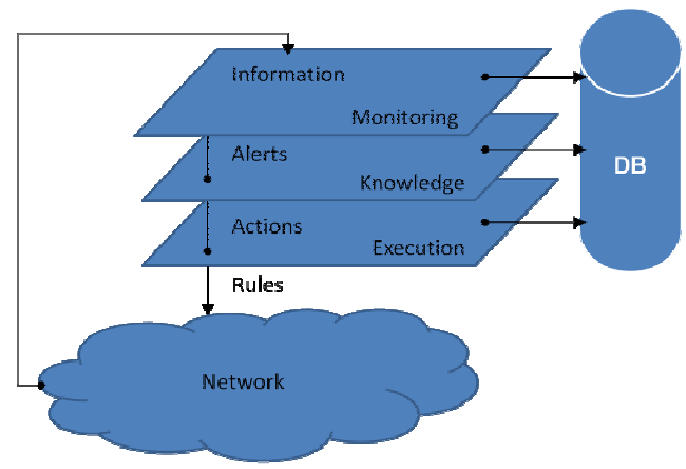

Figure 7. Framework for Selection and Provisioning of Bandwidth Allocation Models

The functional structure of the framework for selection and provisioning of BAM incorporates the basic elements of an autonomic architecture[7][10]. In effect, the framework is capable of applying knowledge based on the network's state (present or past) and then it can define actions (in this case, a BAM selection) to be executed over the network. Therefore, the framework incorporates autonomics characteristics that can be explored on behalf of the implementation of the management plane supporting diverse functionalities.

Regarding the functional modules of the framework, the main function of the Monitoring Module (Figure 7) is to collect relevant network state information and store it at the framework's database. The monitored network state is used by the Knowledge Module for the decision making process regarding BAM option decision and provisioning. Internal actions performed by the Monitoring Model include a standard XML representation of network state and parameters and the analysis of current state in relation to defined SLAs which actually express the user requirements. This module functionality includes, among others, SLA conformance verification, network state evolution identification and alerts generation. 


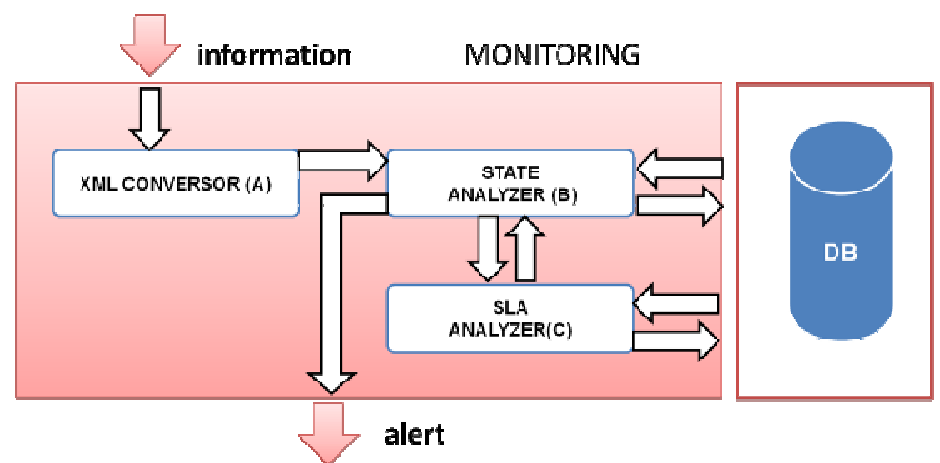

Figure 8. Monitoring Module

The basic functionality of the Knowledge Module (Figure 8) is to introduce autonomic characteristics in the framework in order to replace or minimize, at least partially, the direct human intervention $(\mathrm{G})$. The Knowledge Module deals with the definition of the path selection algorithm (B), the bandwidth allocation model (C), focus of this paper, and other configuration parameters involved. The module also includes an analysis of the network state and verifies the network performance as required by the administrator. The Knowledge Modules intervention at framework level might be trigged through an alert sent by the Monitoring Module or may be programmed to be executed periodically by the network manager. The "actions" over the network computed and defined by this module are executed by the Execution Module.

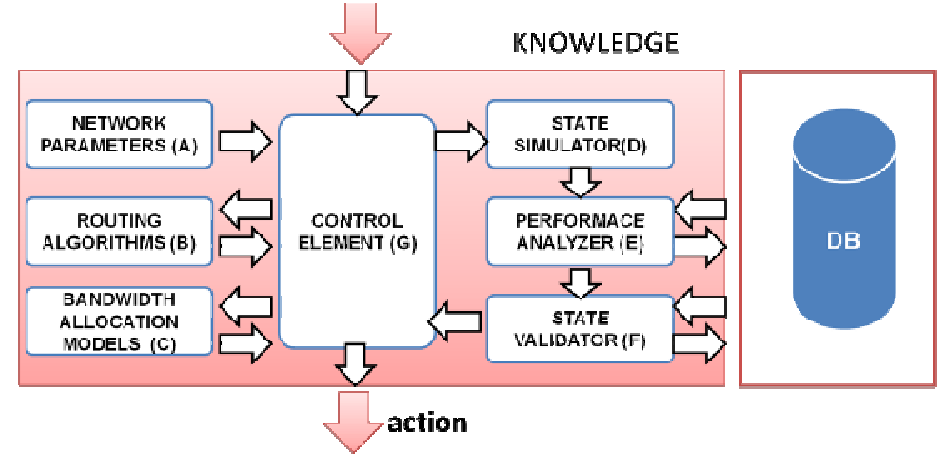

Figure 9. Knowledge Module

The Execution Module, as the name suggests, is responsible by the effective execution of the management control actions over the network and its equipment (Figure 9). In effect, this module maps the defined actions (A) and generates effective rules (B) that are applied (executed) over the network using possibly different deployment mechanisms. The architectural decision for this framework was to adopt OpenFlow as the basic mechanism to enforce rules and actions to the network (section VI). 


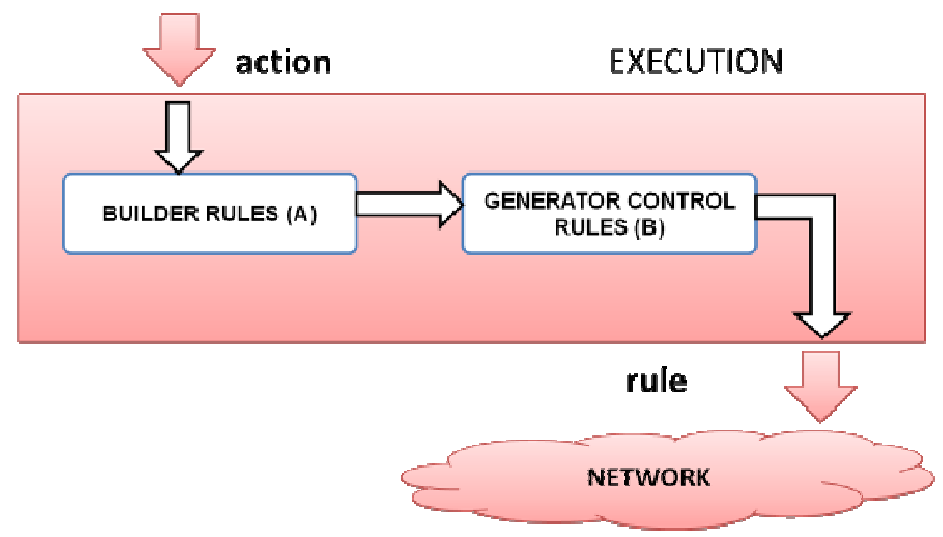

Figure 10. Execution Module.

The framework database module is a persistent repository that stores the network state acquired by the Monitoring Module (established flows, link utilization, other). The database also stores other relevant data for processing such as network topology and configuration parameters such as link capacity, customers SLAs, path selection algorithms, BAM algorithms, network relevant logs and network state history, among others.

\section{FRAMEWORK WITH OPENFLOW DEPLOYMENT}

Software-defined networking (SDN) is a new computational paradigm that enables not only the possibility of developing new network architectures but also supports the solution of specific network and management problems [9].

SDN's architecture adopts fundamentally the separation between control and data plane. The control plane, itself, is programmable by software and, considering network management, allows a more easy abstraction of network's infrastructure due to the centralized approach normally adopted by the OpenFlow controller involved [8].

The incorporation of OpenFlow to the framework was an architectural decision with the main objective to allow centralized and software-defined control of management actions. The deployment is realized through the adoption of a new framework element, the OpenFlow controller (Figure 10) between the framework and managed network elements. In terms of the framework, the rules generated by the Knowledge Module are effectively mapped to OpenFlow flow rules and executed at different equipment used on the network.

The OpenFlow protocol supports the framework operation by intercepting new flow setup requests (flow establishment process). Beyond that, OpenFlow protocol supports all management control functionalities related to network equipment in a centralized way. 


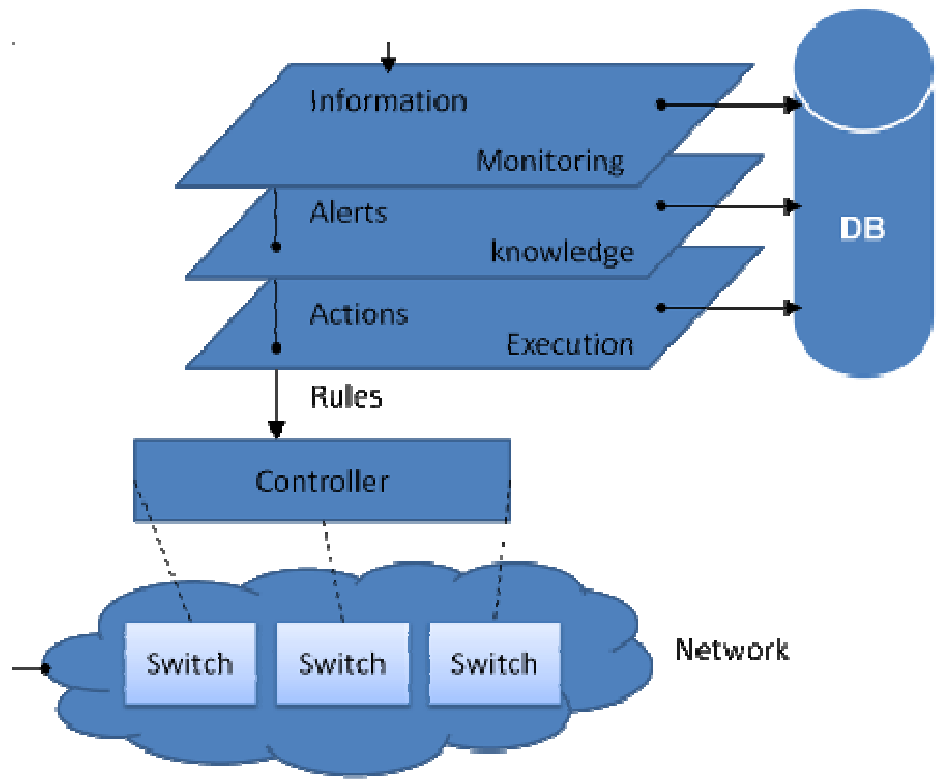

Figure 11. Framework with OpenFlow

\section{BaM Evaluation for Different Traffic Profiles}

This section presents a proof of concept which evaluates the behavior of MAM, RDM and AllocTC-Sharing for different traffic profiles. The objective is to demonstrate that the resulting performance of the network may vary with the utilization of different bandwidth allocation models in different network traffic conditions. As such, it might be recommended to adopt distinct bandwidth allocation models for different network traffic profiles.

Two network traffic profiles (scenarios) with opposite traffic characteristics are evaluated for MAM, RDM and AllocTC-Sharing and the algorithms are compared in relation to their performance.

The performance evaluation used a network topology with one source of traffic (S1) and one destination (D) (Figure 11). CSPF (Constrained Shortest Path First) algorithm was used for path selection.

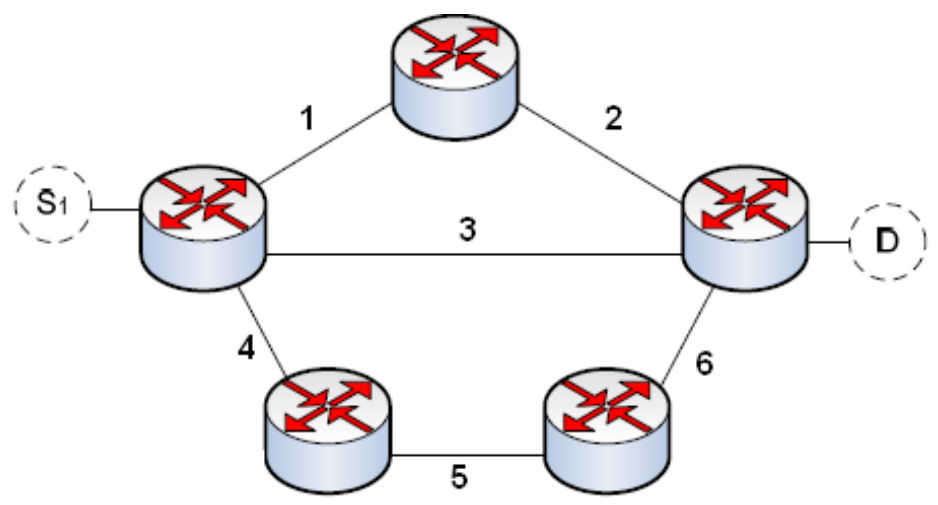

Figure 12. Network Topology - BAM Performance Comparison

Each scenario was simulated with 05 random seeds and the results are presented in terms of the mean value obtained (confidence interval of $95 \%$ ). 
International Journal of Computer Networks \& Communications (IJCNC) Vol.5, No.6, November 2013

The configuration parameters of the simulation scenarios are as follows:

- $\quad$ Links: $622 \mathrm{Mbps}(\mathrm{STM}-4-\mathrm{SDH})$

- $\quad$ Traffic Classes (TCs): TC0, TC1 e TC2

- $\quad$ Bandwidth Constraint (BCs) according Table 2

Table 2. Bandwidth Allocation per Traffic Class (TC)

\begin{tabular}{|l|l|l|l|l|l|l|}
\hline BCs & $\begin{array}{c}\text { Max BC } \\
(\%)\end{array}$ & $\begin{array}{c}\text { MAX BC } \\
(\mathbf{M b p s})\end{array}$ & \multicolumn{1}{|c|}{ TC per BC } & $\begin{array}{c}\text { Max BC } \\
(\%)\end{array}$ & $\begin{array}{c}\text { MAX BC } \\
(\text { Mbps })\end{array}$ & CT per BC \\
\hline & \multicolumn{5}{|c|}{ RDM and AllocTC-Sharing } & \multicolumn{3}{|c|}{ MAM } \\
\hline BC0 & 100 & 622 & TC0+TC1+TC2 & 20 & 124,4 & TC0 \\
\hline BC1 & 80 & 497,6 & TC1+TC2 & 35 & 217,7 & TC1 \\
\hline BC2 & 45 & 279,9 & TC2 & 45 & 279,9 & TC2 \\
\hline
\end{tabular}

In the evaluation scenario 01, the BAM models were compared for a network traffic profile in which there was more traffic (flows) injected in the network for high priority classes (highpriority TCs). In the evaluation scenario 02 , the BAM models were compared in an opposite situation where there was more traffic (flows) for the low-priority classes (low-priority TCs).

The following performance parameters were compared for the BAM models:

- Number of preemptions;

- Number of blocked flows (blocking);

- Number of devolutions; and

- Link utilization per TC.

The basic simulation parameters were:

- Flow - flow setup duration modeled exponentially - mean of 220 seconds; leads to link saturation;

- Flow bandwidth - uniform distribution between 05 Mbps e $25 \mathrm{Mbps}$ and

- Flow number - 1.000

- Flow requests - Inter-arrival interval modeled exponentially with the following values

Table 3. Flow Request Inter-Arrival Interval per Scenario and TC

\begin{tabular}{|l|c|c|}
\hline & Scenario 01 & Scenario 02 \\
\hline TC0 & $1 \mathrm{~s}$ & $3 \mathrm{~s}$ \\
\hline TC1 & $2 \mathrm{~s}$ & $2 \mathrm{~s}$ \\
\hline TC2 & $3 \mathrm{~s}$ & $1 \mathrm{~s}$ \\
\hline
\end{tabular}

It follows an evaluation of the simulation results in relation to each evaluation parameter adopted. 
International Journal of Computer Networks \& Communications (IJCNC) Vol.5, No.6, November 2013

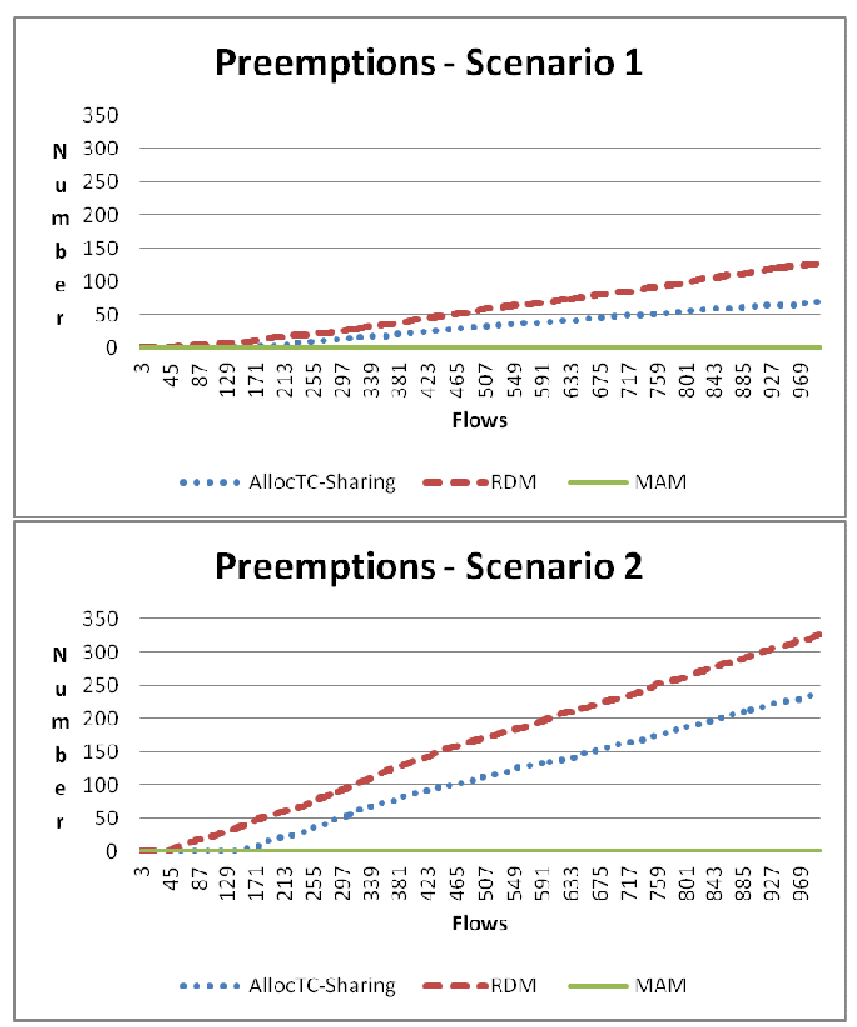

Figure 13. Preemptions

In relation to the number of preemptions resulting from BAM utilization (Figure 12), we observe that the MAM algorithm has the best performance (zero preemption). This result is obvious since MAM does not support any sharing or loan among TCs and, as such, preemptions will never occur. In management terms, this could be a requirement existing in a network. When considering the models RDM and AllocTC-Sharing, which either share or loan bandwidth among TCs, we observe that AllocTC-Sharing generates a smaller number of preemptions for both traffic scenarios. 
International Journal of Computer Networks \& Communications (IJCNC) Vol.5, No.6, November 2013

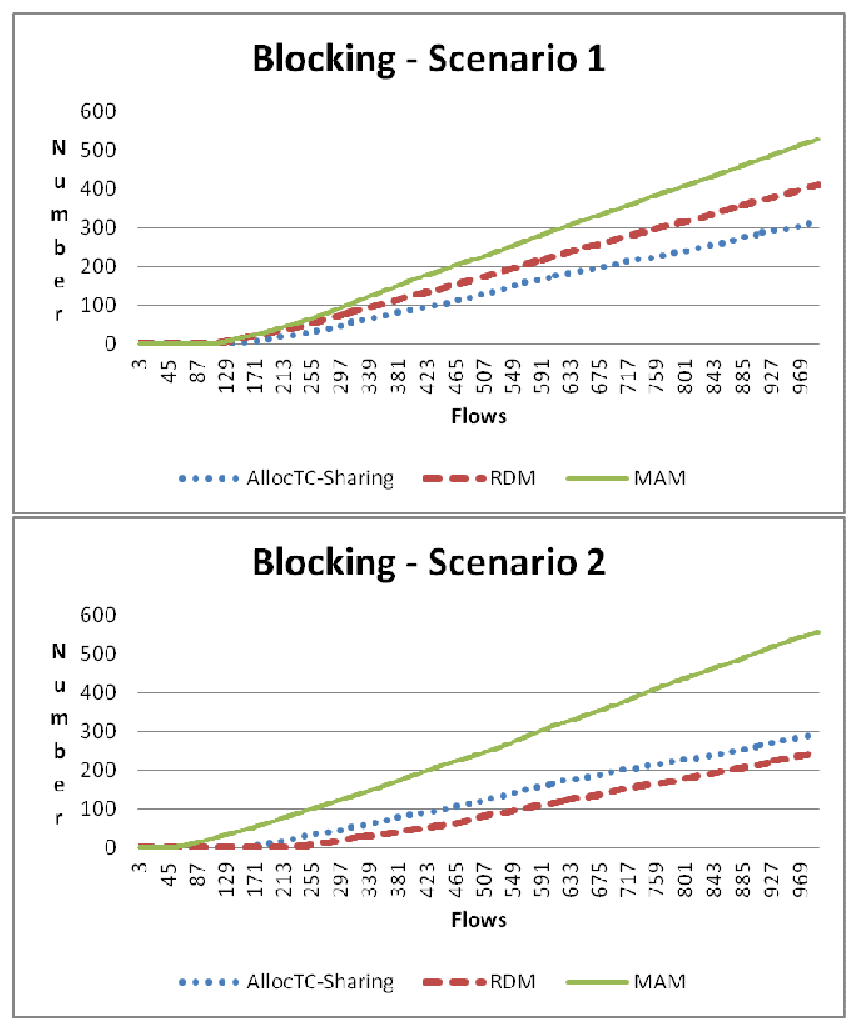

Figure 14. Blocking

In relation to the number of flow setup requests not granted (blocking) (Figure 13), we observe that the MAM algorithm has the worst performance as far as it does not share or loan bandwidth among TCs. In effect, with the increased number of flows setup in the network, the blocking for new flows occurs more frequently. For scenario 01 in which there is a greater number of highpriority flows, we observe a better performance of AllocTC-Sharing model in relation to RDM model. For scenario 02 in which there is a greater number of low-priority flows, we observe that RDM model has a better performance. As such, in a network in which the minimization of blocking could represent a requirement AllocTC-Sharing and RDM could be options for different network scenarios (01 and 02). 
International Journal of Computer Networks \& Communications (IJCNC) Vol.5, No.6, November 2013

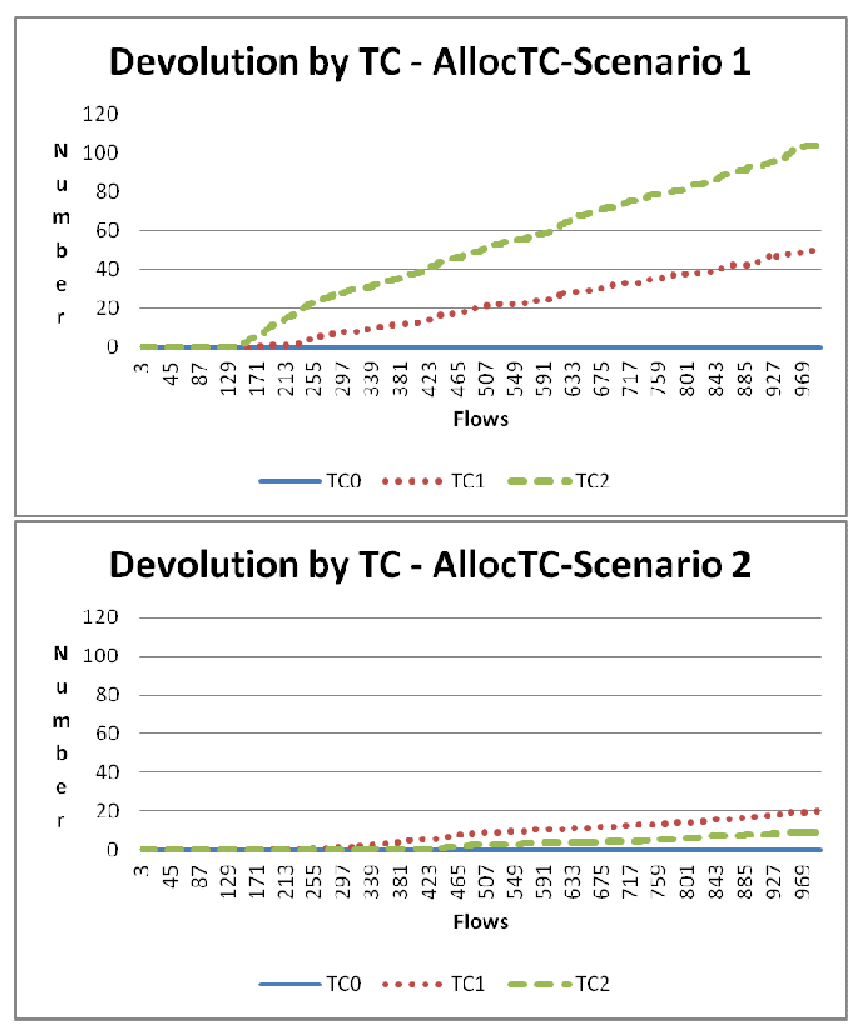

Figure 15. Devolution by TC.

As indicated in section II, the strategy of temporary bandwidth loan from low-priority TCs by high-priority TCs is exclusive of AllocTC-Sharing model. As such, only this model is evaluated in relation to the parameter "bandwidth/ loan devolution" or, in brief, devolution. The evaluation shows that the devolution increases with the number of flows setup for both scenarios. We observe that the scenario 01 has a greater number of "devolutions". In this case, we have a greater number of high-priority flows which take bandwidth from low-priority TCs. As far as the number of established flows increase, leading to link saturation, high-priority flows have to liberate their loans on behalf of low-priority classes. This result shows that the loan strategy providing available low-priority bandwidth for high-priority traffic works better only with a small number of low-priority flows. In case the management does not accept bandwidth devolution, the AllocTC-Sharing is not recommended. 


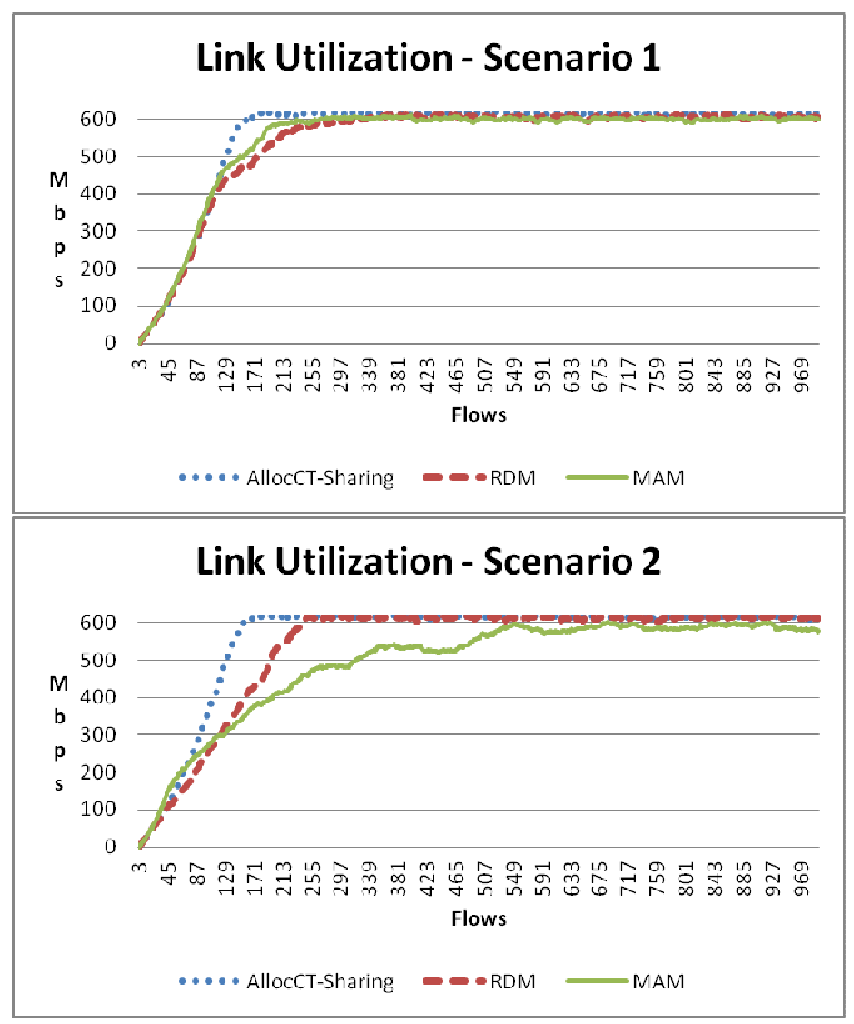

Figure 16. Link utilization

In relation to link utilization (Figure 15), we observe that the AllocTC-Sharing has the best performance and the MAM model presents the worst performance. With the increase of the number of established flows near link and TC saturation, we observe that the models present a similar performance.

\section{CONCLuSion}

The problem of Bandwidth Allocation Model (BAM) provisioning in multiservice networks was addressed in this paper considering the variability of traffic profile inherent for this type of network. A framework under development was presented aiming to support the provisioning of different BAMs for distinct networking traffic scenarios with autonomic and dynamic characteristics. The autonomic characteristic inherent to the framework is intended to improve and facilitate the decision-making process involved with BAM provisioning in multiservice networks.

The framework adopts a centralized management strategy based on OpenFlow in a Software Defined Networking (SDN) implementation style, allowing a uniform and centralized view of the network, its resources and management control actions. Flow rules according with the BAM currently provisioned by the framework are generated and applied to the network by making use of the OpenFlow protocol.

In order to demonstrate the effective need of a OpenFlow-based framework with more dynamic BAM choice, the behaviour of three bandwidth allocation models was evaluated in relation to performance parameters like number of preemptions, flows blocking, bandwidth devolution and link utilization. The obtained results point to the need to allocate distinct BAMs for different network scenarios. 


\section{REFERENCES}

[1] J. Martins, S. Aidarous, T. Plevyak et al, "Managing IP networks: challenges and opportunities", Willey -IEEE Press, September 2003.

[2] Adami, C. Callegari, S. Giordano M. Pagano and M. Toninelli, "G-RDM: a New Bandwidth Constraints Model for DS-TE networks", Proceedings of IEEE GLOBECOM 2007.

[3] Bezerra, R. M. S. ; Martins, J. S. B. . “A Sense and React Plane Structured Autonomic Model Suitable for Quality of Service (QoS) Management". In: 5th International IEEE Workshop on Management of Ubiquitous Communications and Services, 2008, Salvador. 5th International IEEE Workshop on Management of Ubiquitous Communications and Services, 2008.

[4] F. Le Faucheur (a), "Russian dolls bandwidth constraints model for DiffServ-aware MPLS traffic engineering", IETF, RFC 4127, June 2005.

[5] F. Le Faucheur and W. Lai, " Maximum allocation bandwidth constraints model for DiffServ-aware MPLS traffic engineering", IETF, RFC 4125, June 2005.

[6] F. Le Faucheur and W. Lai, "Requirements for support of differentiated services-aware MPLS traffic engineering", IETF, RFC 3564, July 2003.

[7] N. Samaan and A. Karmouch, "Towards autonomic network management: An analysis of current and future research directions," IEEE Communications Surveys Tutorials, vol. 11, no. 3, pp. 22-36, 2009. [Online]. Available: ieeexplore.ieee.org/lpdocs/epic03/wrapper.htm?arnumber=5208731

[8] Nick McKeown, Tom Anderson, Hari Balakrishnan, Guru Parulkar, Larry Peterson, Jennifer Rexford, Scott Shenker, and Jonathan Turner. 2008. "OpenFlow: enabling innovation in campus networks". SIGCOMM Comput. Commun. Rev. 38, 2 (March 2008), 69-74.

[9] Reale, R.F.; da C P Neto, W.; Martins, J. S B, "Routing in DS-TE networks with an opportunistic bandwidth allocation model," Proceedings of the 17th IEEE Symposium on Computers and Communications (ISCC), Cappadocia, Turkey, 2012.

[10] Subharthi Paul, Jianli Pan, and Raj Jain. 2011. "Architectures for the future networks and the next generation Internet: A survey”. Comput. Commun. 34, 1 (January 2011), 2-42.

[11] W. Lai, "Bandwidth constraints models for DiffServ-aware MPLS Traffic Engineering: Performance Evaluation", IETF, RFC 4128, June 2005.

[12] W. Pinto Neto and J. Martins, "A RDM-like Bandwidth Management Algorithm for Traffic Engineering with DiffServ and MPLS Support", Proceedings of the 15th International Conference on Telecommunications - ICT, St. Petersburg, Russia, 2008a.

\section{Authors}

PhD student in Computer Science by DMCC (UFBA/UNIFACS/UEFS), Masters in Computer and Systems by Salvador University - UNIFACS (2011) and graduation in Informatics by Universidade Católica do Salvador (2005). Teaches at Instituto Federal da Bahia - Campus Valença. Has being working in Computer Science with emphasis in computer networks in topics like: Bandwidth Allocation M odel (MAM, RDM, G-RDM, AllocTC-Sharing, ..), MPLS, MPLS-TE, DS-TE, Autonomy, QoS, Future Internet and Software Defined Networks.

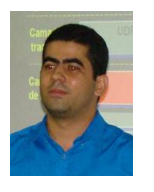

PhD in Computer Science by PMCC (UFBA/UNIFACS/UEFS) (2012), Masters in Computer and Systems by Salvador University - UNIFACS (2005), graduated in Computer Science by Universidade Federal da Bahia (2001). Professor at Instituto Federal de Educação, Ciência e Tecnologia da Bahia (IFBA) and research leader of GSORT - Grupo de Sistemas Distribuídos, Otimização, Redes e Tempo Real (http://www.gsort.ifba.edu.br). Has experience in Computer Science with focus on network management and is involved in security and distance learning projects. Further details at: http://www.romildo.net.

Graduate at Federal University of Paraiba (1977) - Brazil, Masters from Technische Universiteit Eindhoven (TUE)/ NUFFIC (1979) - Holland and Ph.D. from Université Pierre et Marie Curie - UPMC (1986) - France. Has extensive experience in computer science, teaching and researching on innovative areas such as Future Internet, OpenFlow \& Virtualization, Quality of Service, Ubiquitous Com puting and Autonomic Management.

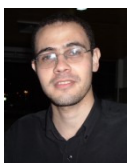
Currently, invited Professor at HTW - Hochschule für Techknik und Wirtschaft des Saarlandes - Germany and Université d’Evry - France. 